\title{
Spatiotemporal characteristics of urban air quality in China and geographic detection of their determinants
}

\author{
ZHANG Xiaoping, GONG Zezhou \\ College of Resources and Environment, University of Chinese Academy of Sciences, Beijing 100049, China
}

\begin{abstract}
Ambient air pollution brought by the rapid economic development and industrial production in China has exerted a significant influence on socio-economic activities and public health, especially in the densely populated urban areas. Therefore, scientific examination of regional variation of urban air quality and its dominant factors is of great importance to regional environmental management. Based on daily air quality index (AQI) datasets spanning from 2014 to 2016, this study analysed the spatiotemporal characteristics of air quality across different regions throughout China and ascertained the determinants of urban air quality in disparate regions. The main findings are as follows: (1) The annual average value of the urban AQI in China decreased from 2014 to 2016, indicating a desirable trend in air quality at the national scale. (2) The attainment rate of the urban AQI exhibited an apparent spatially stratified heterogeneity, wherein North China retained a high AQI value. The increase of Moran's I Index reported an apparent spillover effect among adjacent regions. (3) Both at the national and regional scales, the seasonal tendency of air quality in each year is similar, wherein good in summer and relatively poor in winter. (4) Results drawn from the Geographic Detector analysis show that dominant factors influencing AQI vary significantly across urban agglomerations. Topographical and meteorological variations in urban areas may lead to complex spatiotemporal variations in pollutant concentration. Whereas given the same natural conditions, the human-dominated factors, such as industrial structure and urban form, exert significant impacts on urban air quality. The spatial spillover effects and regional heterogeneity of urban air quality illustrated in this study suggest the governments and institutions should set priority to the importance of regional cooperation and collaboration in light of environment regulation and pollution prevention.
\end{abstract}

Keywords: Air Quality Index (AQI); spatiotemporal characteristics; geographic detector; China

\section{Introduction}

Ambient air pollution resulting from the rapid economic development and industrial production in China has exerted a significant influence on socio-economic activities and public

Received: 2017-05-31 Accepted: 2017-11-18

Foundation: National Natural Science Foundation of China, No.41771133; Science and Technology Service (STS) Program of Chinese Academy of Sciences, No.KFJ-EW-STS-089

Author: Zhang Xiaoping, PhD and Associate Professor, specialized in regional sustainable development. E-mail: zhangxp@ucas.ac.cn 
health, especially in the densely populated urban areas. As a substantial number of diseases have been reported to have a close connection to the severity of air pollution, urban air quality has caught the attention from both governments and individuals (Lelieveld et al., 2015; Li et al., 2015; Chen et al., 2016; Liu et al., 2016; Liu et al., 2017; Qin et al., 2017). To address this concern, the central government of China has adopted a series of new policies to strengthen the environmental management (Hu et al., 2015). In 2012, China's Ministry of Environmental Protection (MEP) issued a new ambient air quality standard (GB 3095-2012), which monitors the concentration of $\mathrm{PM}_{2.5}$, carbon monoxide, sulfur dioxide, nitrogen dioxide, $\mathrm{PM}_{10}$, and ozone in ambient air (MEP, 2012). Considering the diversity of cities throughout the nation, China has employed a three-stage scheme to implement the new ambient air quality standard (Sheng and Tang, 2016). At the end of 2014, 287 prefecture-level cities had implemented this new standard; subsequently, 367 cities began to monitor the six air pollutants and publicize their ambient air quality conditions on the official websites in January of 2015.

Urban air quality in China has attracted much interest from multi-disciplinary perspectives, among which the regional disparity of air pollution is an important one. Most of the studies have explored the spatial spillover effect and regional homogeneity characteristics of air pollution in China from different spatial dimensions, ranging from nationwide to urban agglomerations and specific cities (Sun et al., 2012; Fang et al., 2016; Lin and Wang, 2016; Peng et al., 2016; Gong and Zhang, 2017). To account for the regional variation of air quality, many researchers have examined the influencing factors relevant to urban air quality. In these studies, factors as meteorological elements, land use and land cover change, urbanization, industrialization, energy structure, and transport patterns have been reported to have significant impact on urban air quality (Krummel et al., 1984; Shaw et al., 2010; Tai et al., 2010; Zhang and Fan, 2011; Guan et al., 2014; Lu and Han, 2014; Patton et al., 2014; Li et al., 2015; Lin and Wang, 2016; Qin and Liao, 2016; Sun et al., 2016; Zou et al., 2016; Hu et al., 2017; Yan et al., 2017). The previous studies, in general, have predominantly observed the characteristics of determinants within a single region or among different cities. However, only few of previous researches have explored the driving forces of urban air quality in aspect of regional division and little literature is available so far about the spatially stratified heterogeneity of determinants for AQI in disparate regions throughout China (Zhou et al., 2016). Since regional variation of air quality is complex and its dominant driving factors vary in different regions, comparative analysis at different spatiotemporal scales still requires further research.

To better understand the manner in which air quality and regional determinants interact, we illustrated the spatiotemporal evolution of urban AQI throughout China based on datasets from 2014 to 2016, and explored the influence of relevant factors. Compared with the existing literature, the quantity of sampled cities in our research increased significantly, which helped to engender more accurate and reliable results. Furthermore, the Geographical Detector-based model (Wang, 2010) was used to explore the influence of different geographical factors on urban air quality among disparate regions. Therefore, this research is expected to contribute not only to a further understanding of the current urban air pollution within China but also to a scientific reference for targeted environmental management at regional level. 


\section{Materials and methods}

\subsection{Study area and sample cities}

Due to China's three-stage scheme to implement the new ambient air quality standard, the number of cities with daily AQI data varied significantly within the past few years. Considering the data availability, we selected datasets of 161 sample cities in 2014 and 366 sample cities in both 2015 and 2016 to illustrate the change of overall air quality in China. The location of these cities and their regional division are shown in Figure 1. Most of the cities monitored in 2014 were clustered within relatively more prosperous areas, particularly along the eastern coastal areas. While in 2015 and 2016, the cities monitored in terms of the new standard have spanned to a wide geographical range of Chinese mainland, including $4 \mathrm{mu}-$ nicipalities, 23 provincial capital cities, 5 autonomous region capital cities, 305 prefecture cities (or administrative regions), and 29 county-level cities. Our research is mainly based on the data of 366 sample cities, with 161 sample cities in 2014 as a comparison.

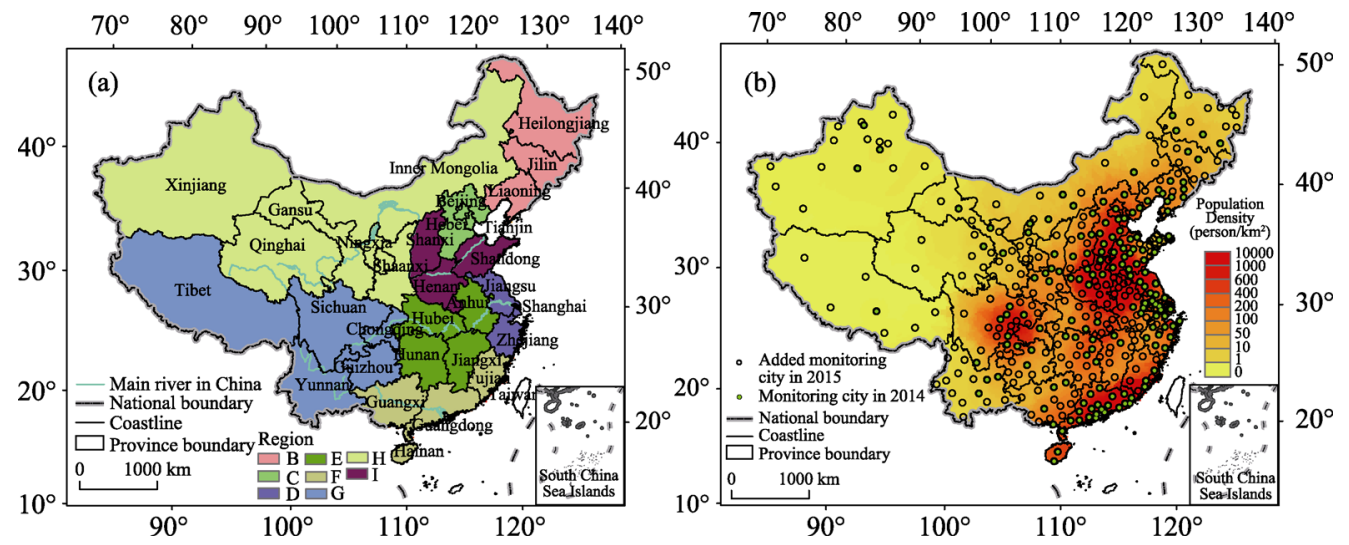

Figure 1 The location of the study area (a) and sample cities (b)

In the Legend of Figure 1a, Region B represents Northeast China, C represents the Beijing-Tianjin-Hebei region, D represents the Yangtze River Delta region, E represents the middle reaches of the Yangtze River, F represents the southeastern coastal areas, G represents Southwest China, H represents Northwest China, and I represents the middle and lower reaches of the Yellow River; see Table 1

\subsection{Data sources}

Daily averaged air quality values for all of the sample cities were downloaded from the official website of China's Ministry of Environmental Protection (http://datacenter.mep.gov.cn). The AQI of 161 cities in 2014 and 366 cities in both 2015 and 2016 were collected. The MEP's AQI is based on Chinese Ambient Air Quality Standards, and is measured as the maximum individual AQI of all pollutants (MEP, 2012). Geographical data of spatial determinants were obtained from the Data Center for Resources and Environmental Sciences, Chinese Academy of Sciences (RESDC) (http://www.resdc.cn). In our Geographical Detector model, 289 sample cities in 2015 were tested due to data availability. Socio-economic data of sample cites were collected from the China Statistics Yearbook of Cities, the China Statistical Yearbook, and relevant provincial or municipal statistical yearbooks. Meteorological data of sample cities were extracted from the interpolation of meteorological data from 824 monitoring stations (http://data.cma.cn). 


\subsection{Methodology}

\subsubsection{Spatial interpolation}

Spatial interpolation, a widely used data estimation method, is based on the proposition that values of a variable in near-by locations are more similar or related than values that are far apart. On the basis of this proposition, spatial interpolation procedure estimates the values of unknown points in an area by computing the data of nearer known points that influence the unknown points (Jeffrey et al., 2001). Given the feature of geographical similarity in China's regional physical geography, we interpolate a raster surface from the points containing related data and then extract the values from the raster for every point of each sample city. The process of Kriging interpolation was conducted in this study. Kriging interpolation is an advanced geostatistical procedure that generates an estimated surface from a scattered set of points, and it can effectively involve an investigation of the spatial manner in which targeted factors interact before the final selection of the best estimation method for generating the output surface (Oliver and Webster, 1990).

\subsubsection{Spatial clusters}

Spatial agglomeration patterns of urban AQI values are acquired by analysing Global Moran's I statistic and employing the Hot Spot detection tool in ArcGIS. The Global Moran's $I$ Index measures the spatial autocorrelation based on both feature locations and feature values simultaneously. The resulting $I$ Index reveals the spatial correlation of the urban AQI: when $I$ is greater than zero, it indicates a positive correlation wherein the urban AQI has an agglomeration pattern; when $I$ is less than zero, it indicates a negative correlation wherein the urban AQI has a diffuse or uniform distribution; and when $I$ is equal to zero, it is uncorrelated, and the urban AQI is distributed irregularly. The Moran's $I$ Index is given as follows (Moran, 1950):

$$
I=\frac{n \sum_{i=1}^{n} \sum_{j=1}^{n} w_{i j}\left(x_{i}-\bar{x}\right)\left(x_{j}-\bar{x}\right)}{\left(\sum_{i=1}^{n} \sum_{j=1}^{n} w_{i j}\right) \sum_{i=1}^{n}\left(x_{i}-\bar{x}\right)^{2}}, \quad(i \neq j)
$$

where $x_{i}$ and $x_{j}$ are the urban AQI of the $i$-th and $j$-th city, respectively, $n$ is equal to the total number of all sample cities, $w_{i j}$ is the spatial weight between the $i$-th and $j$-th city, and $\bar{x}$ is the mean of $x$.

The Hot Spot Analysis tool in ArcGIS operates by comparing each city within the context of neighbouring cities. To be a statistically significant hot spot, a city must possess a high value and be surrounded by other cities with high values as well. The local sum for a city and its neighbours is compared proportionally to the sum of all cities. When the local sum is substantially different from the expected local sum and that difference is too large to be the result of random chance, the resulting statistically significant $Z$ score indicates that a hot spot can be detected accordingly. The Hot Spot Analysis calculates the Getis-Ord $G i^{*}$ statistic for each city in a dataset. By definition, $G i^{*}$ is calculated as follows (Getis and Ord, 1992):

$$
G_{i}^{*}=\frac{\sum_{j=1}^{n} w_{i, j} x_{j}-\bar{x} \sum_{j=1}^{n} w_{i, j}}{S \sqrt{\frac{\left[n \sum_{j=1}^{n} w_{i, j}^{2}-\left(\sum_{j=1}^{n} w_{i, j}\right)^{2}\right]}{n-1}}}
$$

where $w_{i, j}$ is the spatial weight between city $i$ and city $j ; x, n$, and $\bar{x}$ have the same meanings 
as in formula (1), and

$$
S=\sqrt{\frac{\sum_{j=1}^{n} x_{j}^{2}}{n}-(\bar{x})^{2}}
$$

Because $G_{i}^{*}$ is a Z-score, no further calculation is required. The resultant $\mathrm{Z}$ score indicates the location wherein cities with either high or low values will cluster spatially.

\subsubsection{Geographic Detector}

The fundamental theory of the Geographic Detector method was first proposed by Wang et al. (2010) as a tool of detecting and assessing the risks of diseases. The Geographic Detector method used herein is a spatial statistical method employed to test the relationships between geographical phenomena and their potential driving factors (Ju et al., 2016; Wang et al., 2014; Wang et al., 2016). The factor detector, which is a type of Geographic Detector, can quantitatively estimate the relative importance of a particular factor. In this study, the power determinant is defined as the difference between unity and the ratio of the accumulated dispersion variance of the determinants over each sub-region to that over the entire study region (Wang, 2010):

$$
P_{D, U}=1-\frac{1}{n \sigma_{u}^{2}} \sum_{i=1}^{m} n_{D, i} \sigma_{U_{D, i}}^{2}
$$

where $D$ is the influencing factor, $U$ is the affected index, $P_{D, U}$ is the power of determinant, $n$ is the sum of all sample cities, $\sigma_{u}{ }^{2}$ is the variance of the AQI, $m$ is the classification number of an index, and $n_{D, i}$ is the sample number of $\mathrm{D}$ of type $i$. The model is based on the hypothesis wherein $\sigma_{U_{D, i}}^{2} \neq 0$, with $P_{D, U} \in[0,1]$. A significantly larger value of $P_{D, U}$ represents a greater power of influence of a factor, and consequently a higher degree of influence on the urban AQI.

\section{The spatiotemporal evolution of urban air quality}

\subsection{The evolution characteristics of AQI in China}

As shown in Figure 2, the annual average value of the urban AQI in China demonstrates a distinct decrease after 2014. The highest AQI value in 2014 is 235.16 in January, following which it drops to 162.14 in 2015, and thereafter becomes 169.23 in 2016 . It appears that the urban air quality, in general, becomes gradually better from 2014 to 2016. Comparatively, the minimum AQI value exhibits little change, dropping from 52.46 (in 2014) to 50.58 (in 2015), and then to 48.36 (in 2016). These three years have nearly similar variation periods, wherein the air quality is better in summer and autumn, but worse in winter and spring. For the number of attainment days, the summer is better than the other seasons since the AQI values of the majority of days in July, August, and September are lower than 100 (i.e., the attainment standard). However, the higher values (greater than 150) are observed for December, January, and February. Moreover, the range of variation is larger in winter than in summer, and it decreases from 2014 to 2016, which indicates that the air quality in China has become relatively more stable.

In the NAAQS-2012, any daily AQI not exceeding 100 is considered to represent an attainment day. The number of attainment days or the attainment rate during the monitored 


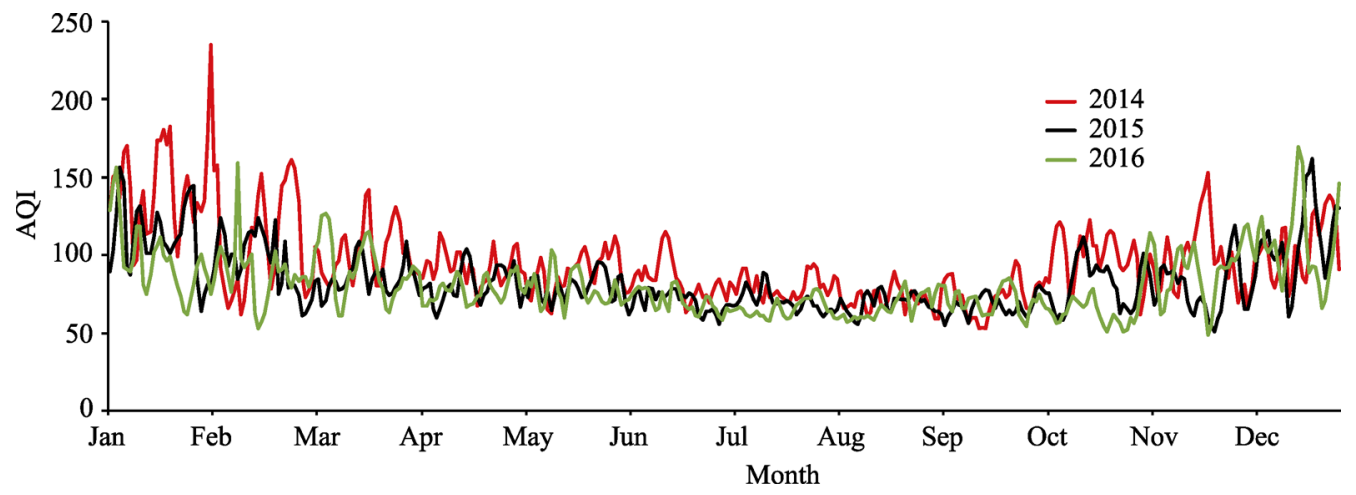

Figure 2 The daily AQI average of sample cities in China in 2014, 2015, and 2016

days is a key index with which to evaluate the air quality of a city. The attainment rate (AR) is the rate during the monitored days when the AQI does not exceed 100, and is computed as follows:

$$
\mathrm{AR}=\frac{\text { Sum of Attainment Days }}{\text { Sum of Total Number of Monitoring Days }}
$$

From the distribution pattern of the attainment rate (Figure 3), it is apparent that urban air quality exhibits a significant agglomeration feature. North China retains the highest values at the nationwide scale, especially in southern Hebei Province, central and western Shandong Province, and central Henan province, where the attainment rates are less than 0.5. Comparatively, cities within the southeastern coastal areas demonstrate significantly different results, for which the attainment rates all exceed 0.7. The contour tool in ArcGIS is used to subdivide the attainment zones (Figure 3). As one of main spatial analysis functions of Arcgis, contour tool creates a line feature class of contours (isolines) from a raster surface. By this tool, regions with similar attainment rate can be separated from other areas. Cities between different layers have distinct values in terms of average attainment rate. It can be observed that cities with lower AR values are clustered in the southern part of North China and the western part of Xinjiang. Accordingly, the AQI of China shows an obvious stratified heterogeneity. In this stratification, the Beijing-Tianjin-Hebei region, the western part of Xinjiang, the west-central part of Shandong, and Henan Province belong to the first layer of attainment, which is characterized by lower attainment values. The middle and lower reaches of the Yangtze River, the middle reaches of the Yellow River, the southern Inner Mongolia, and the areas including north of Hebei, Jilin, and Liaoning belong to the second layer, wherein the attainment rate ranges from 0.3 to 0.7 . The other regions belong to the third

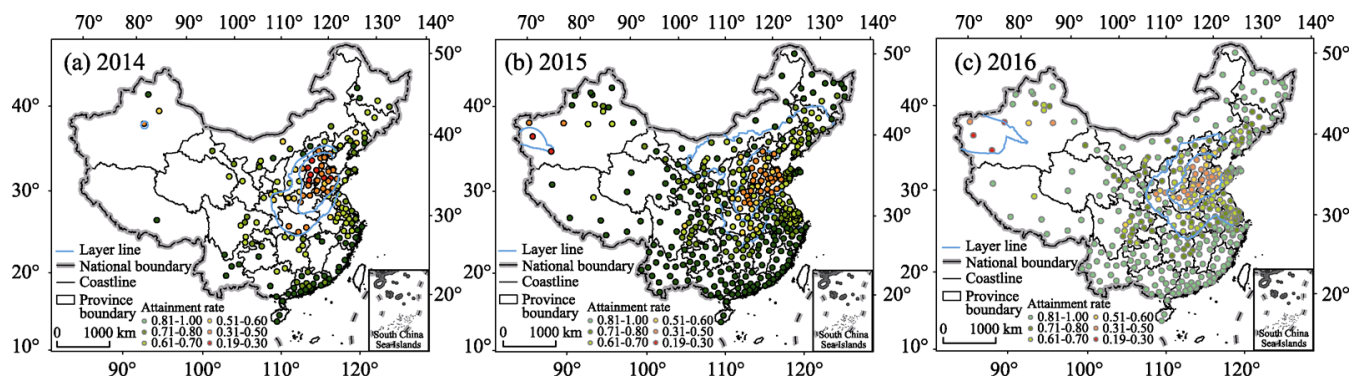

Figure 3 The spatial distribution of the attainment rate for the annually averaged AQI in China 
layer, which demonstrates a relatively good air quality.

Generally, the attainment rate of South China is better than that of North China. The southeastern coastal area and Southwest China retain a better air quality, while the attainment rate of North China exhibits change in some cities from 2014 to 2016. Apparently, the number of attainment cities and attainment days is increasing, which represents a desirable trend.

\subsection{Spatial cluster variation}

Moran's $I$ Index was calculated to test the tendency for spatial clustering (Figure 4). The global Moran's $I$ during the period spanning from 2014 to 2016 is positive, and the spatial pattern of urban AQI displays a positive spatial correlation. The Moran's $I$ in 2014, 2015, and 2016, is $0.695,0.698$, and 0.710 , respectively, which demonstrates a statistically significant increase of spatial clustering trend. Employing a Hot Spot Analysis of the variation in spatial agglomeration of the annual AQI (Figure 5), we discovered the hot spots clustered within the Beijing-Tianjin-Hebei region, the middle and lower reaches of the Yangtze River, and the North China Plain. In addition, some cities in Xinjiang demonstrate an agglomeration of hot spots because of its sandstorm climate. Cold spots are clustered in southern China, including the southeastern coastal areas, Guangdong Province, the Yunnan-Guizhou-Sichuan region, and Heilongjiang Province (which is especially apparent in 2016) (Figure 5c). This suggests an interaction of air quality among cities in adjacent regions. However, the coastal cities along the Yangtze River do not present a significant autocorrelation.
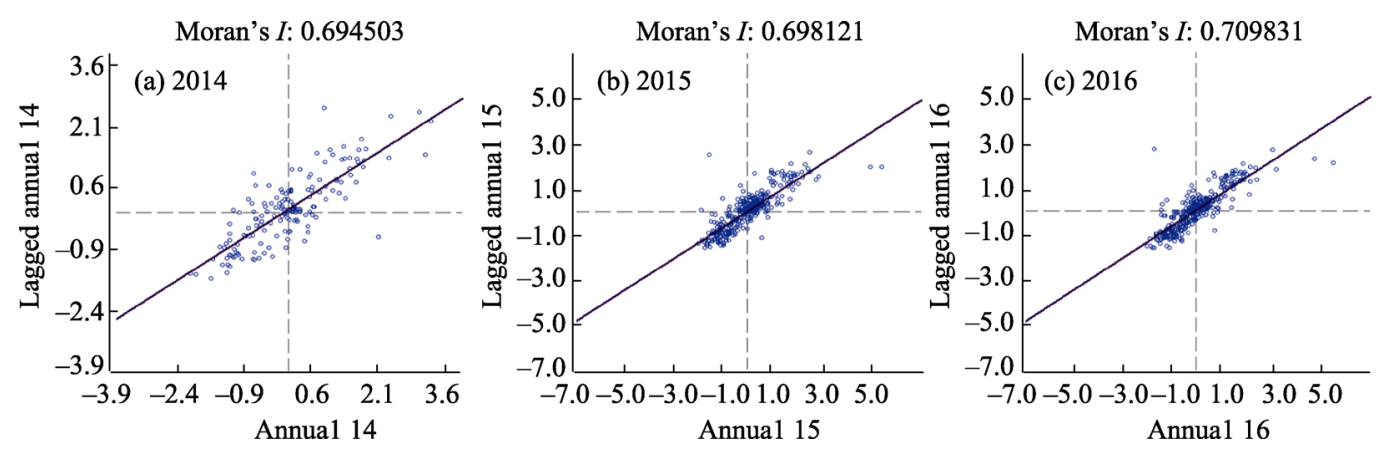

Figure 4 Global autocorrelation of AQI for China in 2014, 2015, and 2016

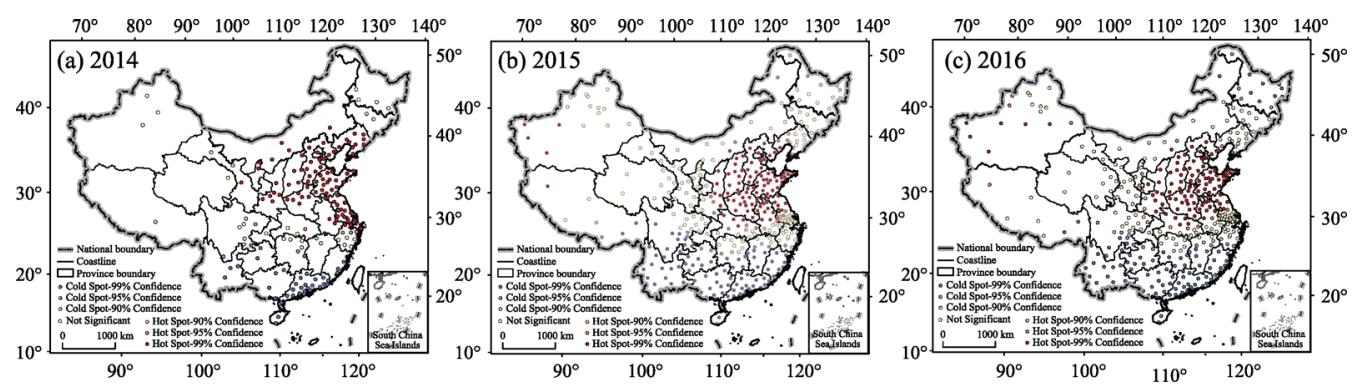

Figure 5 Spatial agglomeration variation of annual AQI in China

\subsection{Comparison among different regions}

To explore the underlying differences and factors of the AQI, we divided the sample cities into eight sub-regions (Figure 1 and Table 1). The principle of our division is to maintain the 
homogeneity within the same region and the heterogeneity among different regions, in which both the physical factors and socio-economic factors are taken into full consideration (Liu et al., 2016; Lin et al., 2016). Specifically speaking, the sub-regions are illustrated as follows. Northeast China is composed of Heilongjiang, Jilin, and Liaoning provinces (Region B). Beijing, Tianjin, and Hebei Province belong to the Beijing-Tianjin-Hebei region (C), which has implemented an integration strategy of environmental management for several years. Shanghai, Jiangsu, and Zhejiang provinces comprise the Yangtze River Delta region (D). Hubei, Hunan, Anhui, and Jiangxi provinces belong to the middle reaches of the Yangtze River (E), while Shandong, Shanxi, and Henan provinces belong to the middle and lower reaches of the Yellow River (I). Considering geographical proximity and climatic similarity, Guangdong, Guangxi, Fujian, and Hainan provinces are classified into the southeastern coastal area (F), which has a relatively better air quality. Sichuan, Guizhou, Yunnan, and Chongqing as well as Tibet belong to Southwest China (G). Finally, Northwest China (H) consists of three provinces as Gansu, Shaanxi, Qinghai, and three autonomous regions as Ningxia, Xinjiang as well as Inner Mongolia. The number of cities in each region is shown in Table 1.

Table 1 Description of regional divisions in China

\begin{tabular}{clcc}
\hline Region & \multicolumn{1}{c}{ Province } & $\begin{array}{c}\text { Number of } \\
\text { city (2014) }\end{array}$ & $\begin{array}{c}\text { Number of city } \\
(2015 \text { and 2016) }\end{array}$ \\
\hline A & Nationwide & 161 & 366 \\
B & Heilongjiang, Jilin, Liaoning & 16 & 37 \\
C & Beijing, Tianjin, Hebei & 13 & 13 \\
D & Shanghai, Jiangsu, Zhejiang & 25 & 39 \\
E & Hubei, Hunan, Anhui, Jiangxi & 14 & 54 \\
F & Guangdong, Guangxi, Fujian, Hainan & 30 & 46 \\
G & Sichuan, Guizhou, Yunnan, Chongqing, Tibet & 15 & 54 \\
H & Gansu, Shaanxi, Qinghai, Ningxia, Xinjiang, Inner Mongolia & 19 & 65 \\
I & Shandong, Shanxi, Henan & 29 & 58 \\
\hline
\end{tabular}

As shown in Figure 6, a significant difference of the urban AQI is observed among regions. The AQI value decreased at the national scale from 2014 to 2015 . The annual average value of the urban AQI was 95.17 in 2014, which decreased further to 82.88 in 2015 and 79.94 in 2016, respectively. Compared with the other regions, the Beijing-Tianjin-Hebei region $(\mathrm{C})$ and the middle and lower reaches of the Yellow River (I) had a higher AQI, while the southeastern coastal areas (F) retained a lower AQI during the three years. The middle reaches of the Yangtze River (E) exhibited the greatest difference between the winter and summer seasons. The Yangtze River Delta region (D) and Southwest China (G) had a similar AQI trend, which remained stable for the whole year except in January and December. The air quality of most cities in China had been improved apparently thereafter 2014. In general, the trend of air quality in each year was similar, both at the national and regional scale, which was good in summer and relatively poor in winter.

\section{Detection of the determinants of $A Q I$ in different regions}

\subsection{Determinants diagram}

Based on the theoretical researches and literature review, we divided factors impacting urban 

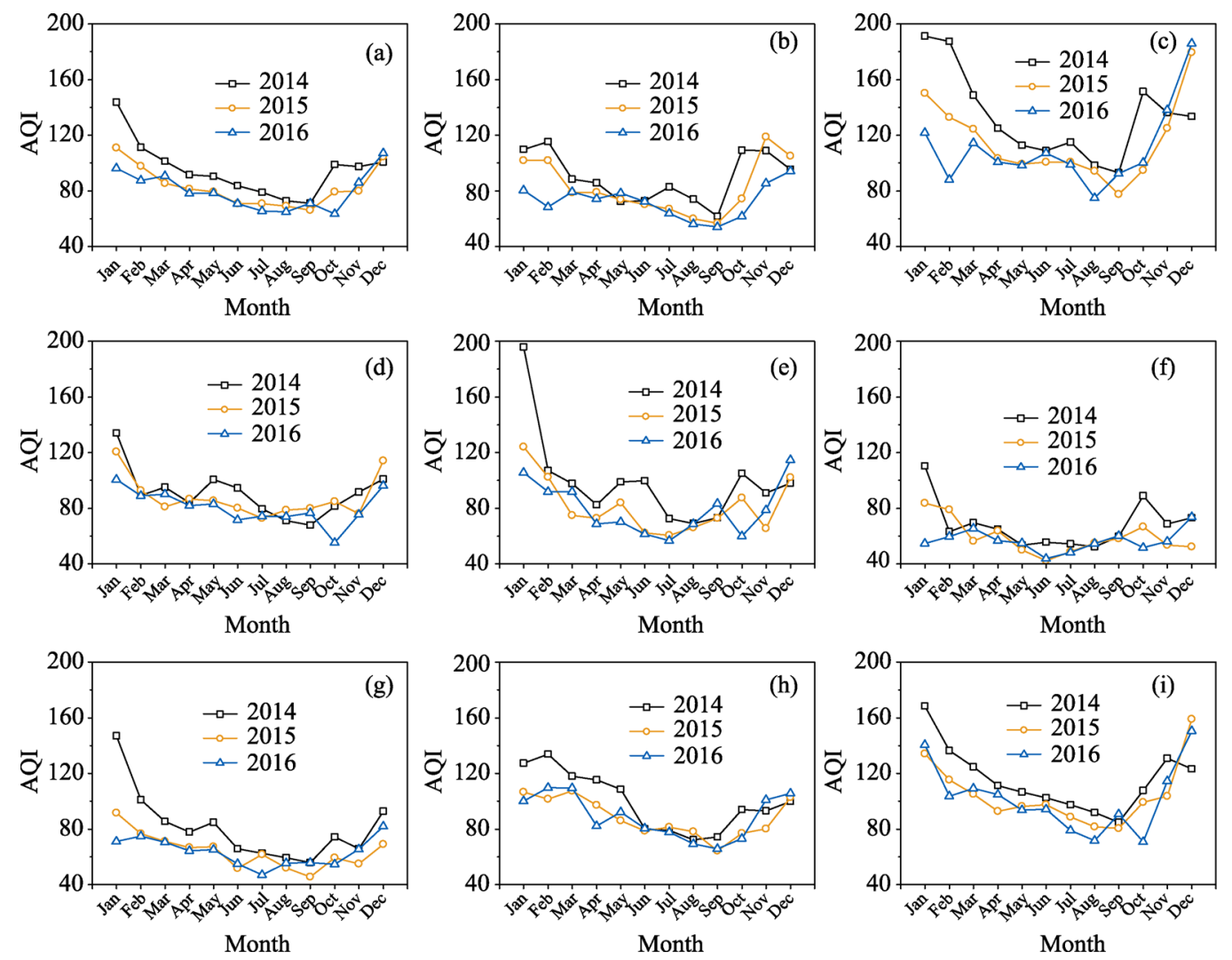

Figure 6 Monthly variations of AQI among different regions of China for 2014, 2015, and 2016

air quality into two main categories: nature-dominated factors and human-dominated factors. Among them, the human-dominated factors are further divided into two sub-groups as urbanization-related factors and industrialization-related factors. Accordingly, we focused on three categories of variables: urbanization, industrial pollution, and natural conditions. A total of 12 indices were selected to provide a comprehensive assessment of the determinants affecting the urban AQI in China, as shown in Figure 7. Urbanization primarily refers to the transformation process experienced by a rural area toward an urban life style, which is represented by an increase of urban population, the expansion of urban built-up areas, and the creation of a landscape and corresponding urban environment with social and life style changes (Morikawa, 1988; Gu et al., 2012). Therefore, when measuring urbanization variables, we selected the annual average population $(X 1)$, the ratio of urban built-up areas to that of the city $(X 2)$, per capita gross domestic product $(X 3)$, and green land area $(X 5)$. Considering industrial production contributes remarkably to air pollution (Place and Mitloehner, 2010), variables consisting of the number of industrial enterprises (X4), industrial dust discharge (X6), and industrial $\mathrm{SO}_{2}$ emission $(X 7)$ are selected to describe industrial pollution. Meanwhile, the natural environment is a fundamental necessity for a region's air quality. Natural condition variables include the slope $(X 8)$, annual average relative humidity $(X 9)$, annual precipitation $(X 10)$, annual average wind speed $(X 11)$, and the annual average temperature $(X 12)$.

Due to data availability, 289 sample cities in 2015 were chosen to investigate the determinants of the urban AQI. All of the above mentioned indices (X1 through X12) were classified into five grades (Table 2) based on a natural break classification and the number of 


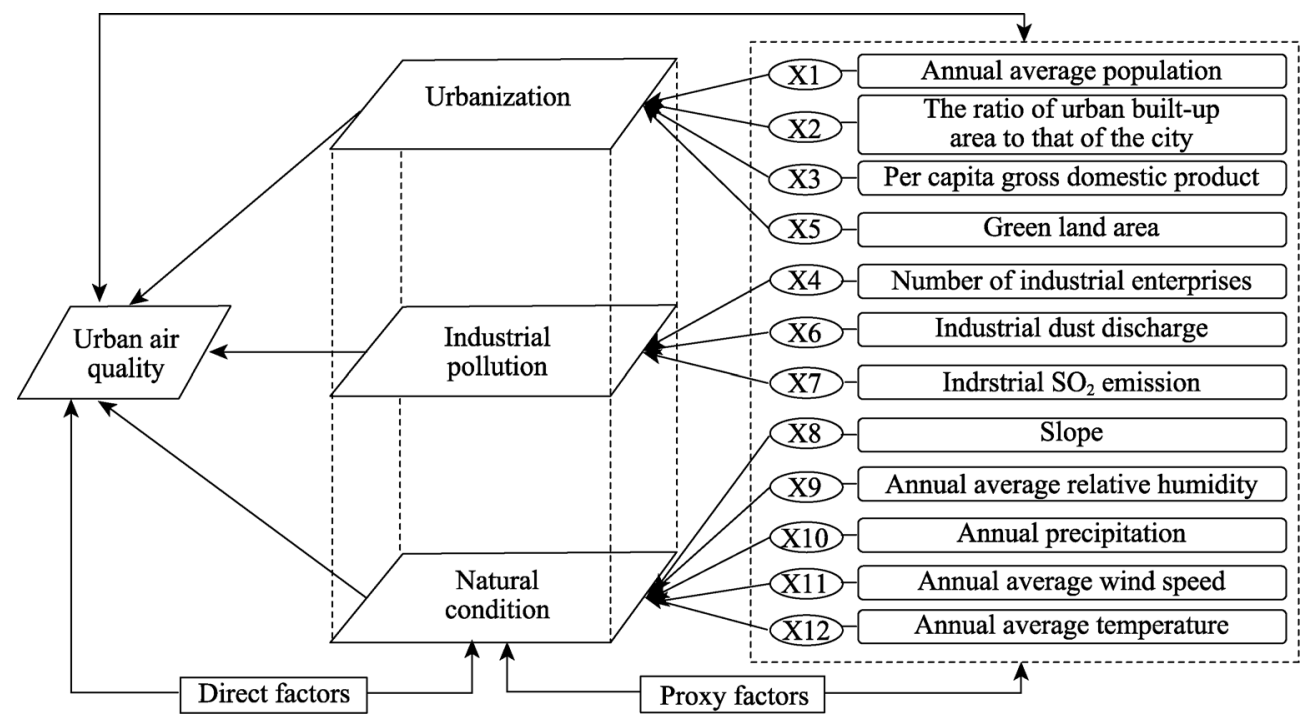

Figure 7 Determinants diagram of urban air quality

same categories within the sample cities. Figure 8 illustrates the spatial distribution and differentiation of the 12 detected factors.

Table 2 Impact factor partitions for the identified geographical factors

\begin{tabular}{lccccc}
\hline \multicolumn{1}{c}{ Indices } & First-grade & Second-grade & Third-grade & Fourth-grade & Fifth-grade \\
\hline$X 1\left(10^{4}\right.$ per $)$ & $<200$ & $200-400$ & $400-700$ & $700-1000$ & $>1000$ \\
$X 2(\%)$ & $<4$ & $4-10$ & $10-18$ & $18-33$ & $>33$ \\
$X 3\left(10^{4}\right.$ yuan $)$ & $<3$ & $3-5$ & $5-7.5$ & $7.5-12$ & $>12$ \\
$X 4\left(10^{2}\right)$ & $<7$ & $7-15$ & $15-25$ & $25-45$ & $>45$ \\
$X 5\left(10^{2} \mathrm{hm}^{2}\right)$ & $<15$ & $15-25$ & $25-40$ & $40-80$ & $>80$ \\
$X 6\left(10^{3} \mathrm{t}\right)$ & $<19$ & $19-23$ & $23-42$ & $42-130$ & $>130$ \\
$X 7\left(10^{4} \mathrm{t}\right)$ & $<3$ & $3-6$ & $6-10$ & $10-16$ & $>16$ \\
$X 8\left({ }^{\circ}\right)$ & $<0.1$ & $0.1-0.5$ & $0.5-1.5$ & $1.5-3.5$ & $>3.5$ \\
$X 9(\%)$ & $<50$ & $50-60$ & $60-70$ & $70-75$ & $>75$ \\
$X 10\left(10^{2} \mathrm{~mm}\right)$ & $<4.5$ & $4.5-7.5$ & $7.5-10$ & $10-15$ & $>15$ \\
$X 11(\mathrm{~m} / \mathrm{s})$ & $<1.4$ & $1.4-1.8$ & $1.8-2.2$ & $2.2-2.6$ & $>2.6$ \\
$X 12\left({ }^{\circ} \mathrm{C}\right)$ & $<7$ & $7-11$ & $11-15$ & $15-19$ & $>19$ \\
\hline
\end{tabular}

\subsection{Results and analysis}

Using the Geographic Detector model, we measured the impact of the 12 factors related to the urban AQI in China. The key findings are as follows:

(1) The significance test of different factors affecting the urban AQI in China (Table 3) reveals that meteorological factors have a more significant influence on AQI compared with other factors. It is also apparent that the annual average temperature has a greater influence relative to other factors except the annual precipitation. In addition, the industrial $\mathrm{SO}_{2}$ emissions indicator is an important factor exerting a greater influence than other socio-economic factors at the national scale.

At the national scale, meteorological conditions and industrial pollution exert obvious impacts on the urban AQI, but the influence of urbanization is limited, which is illustrated from details about region $\mathrm{A}$ in Tables 4 and 5 . The top five factors that influence the 

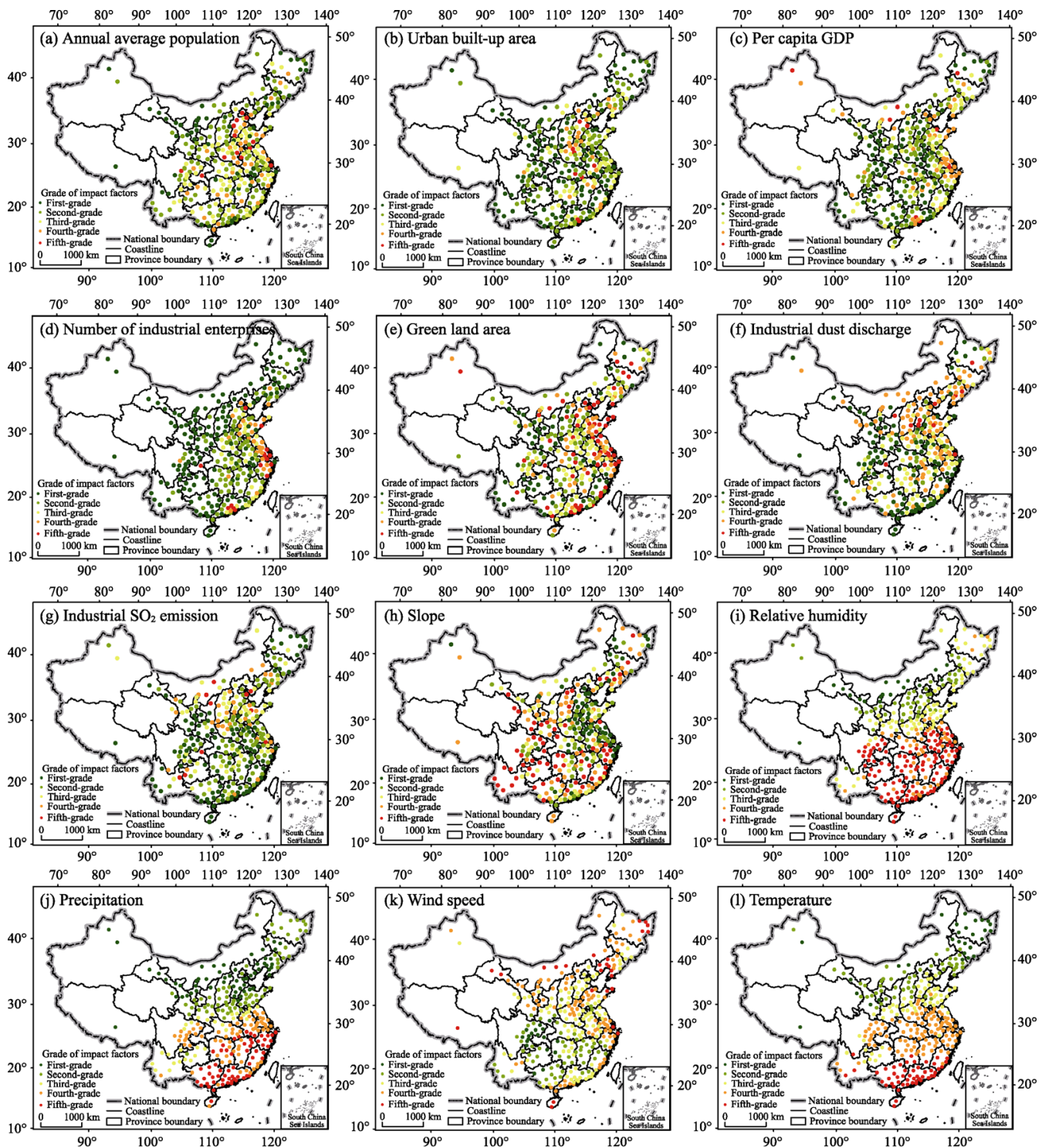

Figure 8 Spatial distribution of the 12 detected factors

Table 3 Significance test of different factors affecting the urban AQI in China (Region A)

\begin{tabular}{|c|c|c|c|c|c|c|c|c|c|c|c|c|}
\hline & $X 1$ & $X 2$ & $X 3$ & $X 4$ & $X 5$ & $X 6$ & $X 7$ & $X 8$ & $\overline{X 9}$ & $X 10$ & $X 11$ & $X 12$ \\
\hline$X 1$ & & & & & & & & & & & & \\
\hline$X 2$ & $\mathrm{~N}$ & & & & & & & & & & & \\
\hline$X 3$ & $\mathrm{~N}$ & $\mathrm{~N}$ & & & & & & & & & & \\
\hline$X 4$ & $\mathrm{~N}$ & $\mathrm{~N}$ & $\mathrm{~N}$ & & & & & & & & & \\
\hline$X 5$ & $\mathrm{~N}$ & $\mathrm{~N}$ & $\mathrm{~N}$ & $\mathrm{~N}$ & & & & & & & & \\
\hline$X 6$ & $\mathrm{~N}$ & $\mathrm{~N}$ & $\mathrm{~N}$ & $\mathrm{~N}$ & $\mathrm{~N}$ & & & & & & & \\
\hline$X 7$ & $\mathrm{~N}$ & $\mathrm{~N}$ & $\mathrm{Y}$ & $\mathrm{N}$ & $\mathrm{N}$ & $\mathrm{N}$ & & & & & & \\
\hline$X 8$ & $\mathrm{~N}$ & $\mathrm{~N}$ & $\mathrm{~N}$ & $\mathrm{~N}$ & $\mathrm{~N}$ & $\mathrm{~N}$ & $\mathrm{~N}$ & & & & & \\
\hline$X 9$ & $\mathrm{~N}$ & $\mathrm{~N}$ & $\mathrm{Y}$ & $\mathrm{N}$ & $\mathrm{N}$ & $\mathrm{N}$ & $\mathrm{N}$ & $\mathrm{N}$ & & & & \\
\hline$X 10$ & $\mathrm{Y}$ & $\mathrm{Y}$ & Y & Y & $\mathrm{Y}$ & $\mathrm{Y}$ & $\mathrm{N}$ & Y & $\mathrm{N}$ & & & \\
\hline$X 11$ & $\mathrm{~N}$ & $\mathrm{~N}$ & $\mathrm{~N}$ & $\mathrm{~N}$ & $\mathrm{~N}$ & $\mathrm{~N}$ & $\mathrm{~N}$ & $\mathrm{~N}$ & $\mathrm{~N}$ & $\mathrm{~N}$ & & \\
\hline$X 12$ & $\mathrm{Y}$ & $\mathrm{Y}$ & $\mathrm{Y}$ & $\mathrm{Y}$ & $\mathrm{Y}$ & $\mathrm{Y}$ & $\mathrm{Y}$ & Y & $\mathrm{Y}$ & $\mathrm{N}$ & $\mathrm{Y}$ & \\
\hline
\end{tabular}


Table 4 Geographically determined weights of the factors affecting the urban AQI in the study area

\begin{tabular}{|c|c|c|c|c|c|c|c|c|c|}
\hline \multirow{2}{*}{ Factors } & \multicolumn{9}{|c|}{$P$ value } \\
\hline & $\mathrm{A}$ & $\mathrm{B}$ & $\mathrm{C}$ & $\mathrm{D}$ & $E$ & $\mathrm{~F}$ & $\mathrm{G}$ & $\mathrm{H}$ & I \\
\hline$X 1$ & 0.1228 & 0.2636 & 0.4115 & 0.2606 & 0.1194 & 0.1440 & 0.2166 & 0.0907 & 0.1765 \\
\hline$X 2$ & 0.1312 & 0.3477 & 0.0736 & 0.1213 & 0.1568 & 0.1759 & 0.1716 & 0.0448 & 0.1071 \\
\hline$X 3$ & 0.0298 & 0.5177 & 0.4911 & 0.1972 & 0.0688 & 0.0830 & 0.1973 & 0.1315 & 0.1514 \\
\hline$X 4$ & 0.1272 & 0.2982 & 0.8172 & 0.5873 & 0.1350 & 0.1104 & 0.1298 & 0.0447 & 0.4137 \\
\hline$X 5$ & 0.1307 & 0.2824 & 0.3109 & 0.2576 & 0.0771 & 0.0421 & 0.5025 & 0.1660 & 0.2235 \\
\hline$X 6$ & 0.1599 & 0.3471 & 0.3368 & 0.4776 & 0.0162 & 0.0134 & 0.0924 & 0.1616 & 0.0491 \\
\hline$X 7$ & 0.1889 & 0.4813 & 0.1442 & 0.3482 & 0.0182 & 0.2016 & 0.0981 & 0.0888 & 0.0450 \\
\hline$X 8$ & 0.1315 & 0.0705 & 0.2278 & 0.1232 & 0.1490 & 0.0442 & 0.4818 & 0.0145 & 0.2327 \\
\hline$X 9$ & 0.2168 & 0.2764 & 0.2746 & 0.2559 & 0.1084 & 0.0067 & 0.3129 & 0.0231 & 0.1483 \\
\hline$X 10$ & 0.2943 & 0.3289 & 0.0397 & 0.6956 & 0.1880 & 0.0592 & 0.4749 & 0.0575 & 0.0148 \\
\hline$X 11$ & 0.1016 & 0.0562 & 0.2641 & 0.2289 & 0.1782 & 0.3815 & 0.4509 & 0.2087 & 0.2351 \\
\hline$X 12$ & 0.3436 & 0.2479 & 0.7128 & 0.0077 & 0.1507 & 0.0450 & 0.3834 & 0.0365 & 0.1590 \\
\hline
\end{tabular}

Table 5 The top three factors affecting the urban AQI for individual regions

\begin{tabular}{ccccccc}
\hline Region & Factors & $P$ value & Factors & $P$ value & Factors & $P$ value \\
\hline A & $X 12$ & 0.3436 & $X 10$ & 0.2943 & $X 9$ & 0.2168 \\
B & $X 3$ & 0.5177 & $X 7$ & 0.4813 & $X 2$ & 0.3477 \\
C & $X 4$ & 0.8172 & $X 12$ & 0.7128 & $X 3$ & 0.4911 \\
D & $X 10$ & 0.6956 & $X 4$ & 0.5873 & $X 6$ & 0.4776 \\
E & $X 10$ & 0.1880 & $X 11$ & 0.1782 & $X 2$ & 0.1568 \\
F & $X 11$ & 0.3815 & $X 7$ & 0.2016 & $X 2$ & 0.1759 \\
G & $X 5$ & 0.5025 & $X 8$ & 0.4818 & $X 10$ & 0.4749 \\
H & $X 11$ & 0.2087 & $X 5$ & 0.1660 & $X 6$ & 0.1616 \\
I & $X 4$ & 0.4137 & $X 11$ & 0.2351 & $X 8$ & 0.2327 \\
\hline
\end{tabular}

urban AQI are the annual average temperature (0.3436), annual precipitation (0.2943), annual average relative humidity (0.2168), industrial $\mathrm{SO}_{2}$ emission (0.1889), and industrial dust discharge (0.1599). For most regions, the $P$ values for annual precipitation remained high, especially for the Yangtze River Delta region (D) and Southwest China (G) where the $P$ values for annual precipitation were 0.6956 and 0.4749 , respectively, which indicates that annual precipitation plays a key role in maintaining good air quality.

(2) Disparate regions are characterized by different primary determinants (Tables 4 and 5). The dominant factors for the urban AQI in Northeast China (B) are the per capita GDP (0.5177), industrial $\mathrm{SO}_{2}$ emission (0.4813), and the ratio of urban built-up areas (0.3477). This reveals that industrial production and the economic development of Northeast China have a fundamental influence on its air quality. Meanwhile, the influences of precipitation (0.3289) and temperature (0.2479) are lower than those of industrial factors.

The dominant factors in the Beijing-Tianjin-Hebei region $(\mathrm{C})$ are the number of industrial enterprises (0.8172), annual average temperature (0.7128), per capita GDP (0.4911), and annual average population (0.4115). The number of industrial enterprises represents the extent of air pollution, and has a significant correlation with the AQI. Temperature influences air flow and diffusion, which have a significant impact on air quality. The GDP reflects the industrial development and urbanization of a region, and therefore has a certain relationship with air quality. Meanwhile, population is a key factor for the Beijing-Tianjin-Hebei region, as the cluster of population in this region is directly correlated with pollutant emissions from vehicles and households, etc. This result supports the hypothesis that population is a key 
influencing factor on the air quality in densely populated areas, same as other researchers have argued that anthropogenic emissions impact the regional climate and air quality (Chen et al., 2017; Li et al., 2017).

The dominating factors in the Yangtze River Delta region (D) are annual precipitation (0.6956), the number of industrial enterprises (0.5873), and industrial dust discharge (0.4776). Since the Yangtze River Delta is adjacent to the sea, precipitation has a substantial influence on the urban AQI. The frequent rainfall in the region significantly improves the urban air quality, and industrial production has a greater influence than other factors since it represents a direct source of air pollution.

The foremost factors in the middle reaches of the Yangtze River (E) are annual precipitation (0.1880), annual average wind speed (0.1782), and the ratio of urban built-up areas (0.1568). These factors reflect that precipitation plays an important role in improving the air quality, while wind speed affects the diffusion of air pollution. Compared with other regions, these three factors have a lower influence on air quality in this region.

The principal factors for the southeastern coastal areas (F) are the annual average wind speed (0.3815), the industrial $\mathrm{SO}_{2}$ emissions (0.2016), the ratio of urban built-up areas $(0.1759)$, and the annual average population $(0.1440)$. The significance of wind speed factor demonstrates that coastal weather and the corresponding air circulation greatly affect air quality in southeastern China, the reason for which is that, under tropical and subtropical monsoon climates, easterly and southeasterly winds are prevalent during spring (March to May) and southwesterly winds are prevalent during midsummer (June to August). Since these winds blow clean air from the sea to the coast, it is helpful to improve the air quality (Sheng and Tang, 2016). Moreover, industrial pollution has a direct influence on air quality, while the flow of pollution is a significant factor as well. Finally, since many people are clustered in the southeastern coastal areas, especially in the Pearl River Delta, the size of the population also has a significant influence on the air quality within this region.

The prevailing factors in Southwest China (G) are the green land area (0.5025), slope (0.4818), and annual precipitation (0.4749). This suggests that topography has a significant influence on the AQI in Southwest China, especially in the Sichuan Basin, since the slope has a notable influence on air diffusion and circulation. Moreover, the area of green land and forest coverage within an urban territory has a positive influence on the improvement of urban air quality.

The dominating factors in Northwest China $(\mathrm{H})$ are the annual average wind speed (0.2087), area of green land (0.1660), industrial dust discharge (0.1616), and the per capita GDP (0.1315). Compared with other regions, economic development and industrial production tend to play a more important role in air quality in Northwest China.

The dominant factors in the middle and lower reaches of the Yellow River (I) are the number of industrial enterprises (0.4137), annual average wind speed (0.2351), and slope (0.2327). Industrial emission is the key factor for this region, and it is thus necessary to implement regional joint control to improve air quality. Wind speeds and slope also affect the air circulation in this region.

(3) There are obvious differences among the 12 examined factors. The urbanization factors have a greater influence on air quality in metropolis areas, especially the factor $X 1$ (annual average population) whose $P$ value is higher in the Beijing-Tianjin-Hebei and Yangtze River Delta regions. The factor $X 3$ (per capita GDP) has greater influence in Northeast 
China, the Beijing-Tianjin-Hebei and Yangtze River Delta regions. The factor X5 (green land area) exerts a more powerful influence in Southwest China, the Beijing-Tianjin-Hebei region as well as Northeast China. Meanwhile, the number of industrial enterprises $(X 4)$ has the greatest influence $(0.8172)$ relative to other factors in the Beijing-Tianjin-Hebei region, by contrast with its weak influence on AQI in Northwest China (0.0447). Generally, the industrial factors possess greater significance in regions which are characterized by a more developed industry, such as the Beijing-Tianjin-Hebei and Yangtze River Delta regions. Meteorological conditions influence nearly all of the regions, while the slope only matters in regions with wide-range topographic variations. Annual precipitation and annual average relative humidity have more influence in the Yangtze River Delta region and South China, while the annual average wind speed matters more substantially in South China. The annual average temperature has the greatest influence on AQI in the Beijing-Tianjin-Hebei region (0.7128) compared with the other regions.

\section{Conclusions and implications}

\subsection{Conclusions}

Based on a dataset spanning from 2014 to 2016, our study analysed the spatiotemporal patterns of the urban AQI in China in terms of attainment rates, seasonal differentiation, and regional divisions. Furthermore, we focused on exploring the geographical determinants affecting AQI among different regions by combining the GIS spatial analysis and the Geographic Detector method. The following main conclusions can be drawn from the study:

(1) The annual average value of the urban AQI in China decreased from 2014 to 2016, representing a desirable trend of air quality. However, the worst air quality days still appeared during winter, while the air quality in summer was relatively good and stable.

(2) The attainment rate of the urban AQI exhibits an apparently spatial stratified heterogeneity. The south-central region of North China and the western part of Xinjiang retained the worst air quality at the national scale. Cities with medium grade of air quality are mainly located in the middle and lower reaches of the Yangtze River, the middle reaches of the Yellow River, southern Inner Mongolia, and the northern part of Hebei, Jilin, and Liaoning provinces. Other regions demonstrated a good attainment rate of the average annual air quality.

(3) The increase of Moran's I Index of the urban AQI from 2014 to 2016 demonstrates a statistically significant increase of spatial clustering trend, which indicates that urban air quality exhibits an apparent agglomeration feature. Hot spots were reported in the Beijing-Tianjin-Hebei region, the middle and lower reaches of the Yangtze River, and North China. Meanwhile, a major cold spot was clustered in Southern China. However, the coastal cities along the Yangtze River did not possess any significant autocorrelation.

(4) Both at the national and regional scale, the tendency of air quality in each year is similar, i.e., good values in summer and relatively poor values in winter. Compared with the other regions, the Beijing-Tianjin-Hebei region and the middle and lower reaches of the Yellow River maintained higher AQI values over each of the three years. Meanwhile, the southeastern coastal areas retained a lower AQI for the three years, and the middle reaches of the Yangtze River exhibited the greatest differences between winter and summer. 
(5) Disparate regions have different determinants for the urban AQI. Meteorological conditions and industrial pollution exert obvious impacts on the urban AQI, but the influence of urbanization is limited from the national scale. Generally, industrial factors have greater influence in regions which possess higher levels of industrialization. Meteorological conditions play an important role toward improving air quality, while the slope affects air diffusion in regions with high topographic variations.

\subsection{Implications}

The findings in our paper have significant policy implications. Air quality management plan has been employed by Chinese government as the most important one of the series of policies in managing urban air quality. The successful implementation of air quality policies relies on multi-factors existing at different scales, e.g. national, municipal and local. Undeniably, topographical and meteorological variations in urban areas, which are generally beyond our control, may lead to complex spatiotemporal variation in pollutant concentration. Whereas given the same natural conditions, the human-dominated factors, such as industrial structure and urban form which exert significant impacts on urban air quality are within the control of human activities. Thus, optimizing industrialization and urbanization initiatives and strengthening their environmental implications should be the key points to improve urban air quality. Furthermore, the regional heterogeneity of urban air quality urges Chinese municipal governments strengthen regional cooperation and deepen bilateral collaboration in light of air regulation and pollution prevention.

Due to data limitations, the long-term change of urban air quality has not been deeply discussed in this study. Meanwhile, the complexity of urban air quality and its interaction mechanism with influencing factors still remain to be studied in the future. We need to accumulate more data and to explore new avenues to research the spatiotemporal variations of urban air quality and its driving forces.

\section{References}

Chen J, Li C, Ristovski Z et al., 2017. A review of biomass burning: Emissions and impacts on air quality, health and climate in China. Science of The Total Environment, 579: 1000-1034.

Chen X, Zhang LW, Huang J J et al., 2016. Long-term exposure to urban air pollution and lung cancer mortality: A 12-year cohort study in Northern China. Science of The Total Environment, 571: 855-861.

Fang C L, Wang Z B, Xu G, 2016. Spatial-temporal characteristics of $\mathrm{PM}_{2.5}$ in China: A city-level perspective analysis. Journal of Geographical Sciences, 26(11): 1519-1532.

Getis A, Ord J K, 1992. The analysis of spatial association by use of distance statistics. Geographical Analysis, 24(3): 189-206.

Gong Z Z, Zhang X P, 2017. Assessment of urban air pollution and spatial spillover effects in China: Cases of 113 key environmental protection cities. Journal of Resources and Ecology, 8(6): 584-594.

$\mathrm{Gu}$ C, Wu L, Lan C, 2012. Progress in research on Chinese urbanization. Frontiers of Architectural Research, 1(2): 101-149.

Guan D, Su X, Zhang Q et al., 2014. The socioeconomic drivers of China's primary $\mathrm{PM}_{2.5}$ emissions. Environmental Research Letters, 9(2): 024010.

Hu J, Huang L, Chen M et al., 2017. Impacts of power generation on air quality in China (Part II): Future scenarios. Resources Conservation \& Recycling, 121: 115-127.

$\mathrm{Hu}$ J, Ying Q, Wang Y et al., 2015. Characterizing multi-pollutant air pollution in China: Comparison of three air quality indices. Environment International, 84: 17-25.

Jeffrey S J, Carter J O, Moodie K B et al., 2001. Using spatial interpolation to construct a comprehensive archive of Australian climate data. Environmental Modelling \& Software, 16(4): 309-330.

$\mathrm{Ju} \mathrm{H}$, Zhang Z, Zuo L et al., 2016. Driving forces and their interactions of built-up land expansion based on the geographical detector: A case study of Beijing, China. International Journal of Geographical Information 
Science, 30(11): 1-20.

Krummel J R, Gilmore C C, O'Neill R V, 1984. Locating vegetation at-risk to air pollution: An exploration of a regional approach. Journal of Environmental Management, 18(3): 279-290.

Lelieveld J, Evans J S, Fnais M et al., 2015. The contribution of outdoor air pollution sources to premature mortality on a global scale. Nature, 525(7569): 367-371.

Li P, Yan R, Yu S et al., 2015. Reinstate regional transport of $\mathrm{PM}_{2.5}$ as a major cause of severe haze in Beijing. Proceedings of the National Academy of Sciences of the United States of America, 112(21): 2739-2740.

Li Q, Jiang J, Wang S et al., 2017. Impacts of household coal and biomass combustion on indoor and ambient air quality in China: Current status and implication. Science of The Total Environment, 576: 347-361.

Lin X, Wang D, 2016. Spatiotemporal evolution of urban air quality and socioeconomic driving forces in China. Journal of Geographical Sciences, 26(11): 1533-1549.

Liu C, Chen R, Zhao Y et al., 2017. Associations between ambient fine particulate air pollution and hypertension: A nationwide cross-sectional study in China. Science of The Total Environment, 584/585: 869-874.

Liu W, Cai J, Huang C et al., 2016. Associations of gestational and early life exposures to ambient air pollution with childhood atopic eczema in Shanghai, China. Science of The Total Environment, 572: 34-42.

Lu X, Han L, 2014. Research on meteorological features of PBL during heavy haze episodes in the city of Chengdu, Sichuan basin, China. American Geographical Union, Fall Meeting.

Ministry of Environmental Protection (MEP) of China, 2012. Ambient Air Quality Standards GB3095-2012. Beijing: China's Ministry of Environmental Protection. (in Chinese)

Moran P A P, 1950. Notes on continuous stochastic phenomena. Biometrika, 37(1/2): 17-23.

Morikawa H, 1988. On the phenomenon of population turnaround or "counterurbanization". Geographical Review of Japan, 61: 685-705.

Oliver M A, Webster R, 1990. Kriging: A method of interpolation for geographical information systems. International Journal of Geographical Information Science, 4(3): 313-332.

Patton A P, Perkins J, Zamore W et al., 2014. Spatial and temporal differences in traffic-related air pollution in three urban neighborhoods near an interstate highway. Atmospheric Environment, 99: 309-321.

Peng J, Chen S, Lü H et al., 2016. Spatiotemporal patterns of remotely sensed $\mathrm{PM}_{2.5}$ concentration in China from 1999 to 2011. Remote Sensing of Environment, 174: 109-121.

Place S E, Mitloehner F M, 2010. Invited review: Contemporary environmental issues: A review of the dairy industry's role in climate change and air quality and the potential of mitigation through improved production efficiency. Journal of Dairy Science, 93(8): 3407-3416.

Qin H, Liao T F, 2016. The association between rural-urban migration flows and urban air quality in China. Regional Environmental Change, 16(5): 1-13.

Qin R X, Xiao C, Zhu Y et al., 2017. The interactive effects between high temperature and air pollution on mortality: A time-series analysis in Hefei, China. Science of The Total Environment, 575: 1530-1537.

Shaw D, Pang A, Lin C C et al., 2010. Economic growth and air quality in China. Environmental Economics and Policy Studies, 12(3): 79-96.

Sheng N, Tang U W, 2016. The first official city ranking by air quality in China: A review and analysis. Cities, 51: 139-149.

Sun D, Du W P, Gao Q X et al., 2012. Change characteristics of API of several typical cities within three urban agglomerations in China from 2001 to 2010. Resources Science, 34(8): 1401-1407. (in Chinese)

Sun L, Wei J, Duan D H et al., 2016. Impact of land-use and land-cover change on urban air quality in representative cities of China. Journal of Atmospheric and Solar-Terrestrial Physics, 142: 43-54.

Tai A P K, Mickley L J, Jacob D J, 2010. Correlations between fine particulate matter $\left(\mathrm{PM}_{2.5}\right)$ and meteorological variables in the United States: Implications for the sensitivity of $\mathrm{PM}_{2.5}$ to climate change. Atmospheric Environment, 44(32): 3976-3984.

Wang J F, 2010. Spatial Analysis. Beijing: Science Press, 50-61. (in Chinese)

Wang J F, Ge Y, Li L et al., 2014. Spatiotemporal data analysis in geography. Acta Geographica Sinica, 69(9): 1326-1345. (in Chinese)

Wang J F, Li X H, Christakos G et al., 2010. Geographical detectors-based health risk assessment and its application in the neural tube defects study of the Heshun region, China. International Journal of Geographical Information Science, 24(1): 107-127.

Wang J F, Zhang T L, Fu B J, 2016. A measure of spatial stratified heterogeneity. Ecological Indicators, 67: $250-256$.

Yan Z, Xin Y, Brown R et al., 2017. Shipping emissions and their impacts on air quality in China. Science of the Total Environment, 581/582: 186-198.

Zhang R, Fan S, 2011. Study of the influence of wind field on air quality over the Pearl River Delta. Acta Scientiarum Natralium Universitatis Sunyatseni, 50(6): 130-134. (in Chinese)

Zhou L, Wu J, Jia R et al., 2016. Investigation of temporal-spatial characteristics and underlying risk factors of $\mathrm{PM}_{2.5}$ pollution in Beijing-Tianjin-Hebei area. Research of Environmental Sciences, 29(4): 483-493. (in Chinese)

Zou B, Xu S, Sternberg T et al., 2016. Effect of land use and cover change on air quality in urban sprawl. Sustainability, 8(7): 677-690. 